\title{
The Composition and Structure of White Dwarf Atmospheres Revealed by Extreme Ultraviolet Spectroscopy
}

\section{MARTIN A. BARSTOW, ${ }^{1}$ IVAN HUBENY, ${ }^{2}$ THIERRY LANZ, ${ }^{2}$ JAY B. HOLBERG, ${ }^{3}$ AND EDWARD M. SION ${ }^{4}$}

\author{
${ }^{1}$ Department of Physics and Astronomy, University of Leicester, \\ University Road, Leicester LE1 7RH, UK \\ ${ }^{2}$ Universities Space Research Association NASA/GSFC, Greenbelt, MA 20711, USA \\ ${ }^{3}$ Lunar and Planetary Laboratory, University of Arizona, Tucson, AZ 85721, USA \\ 4 Department of Astronomy and Astrophysics, Villanova University, Villanova, PA 19085, USA
}

The ROSAT and EUVE all-sky surveys have resulted in an important change in our understanding of the general composition of hydrogen-rich DA white dwarf atmospheres, with the photospheric opacity dominated by heavy elements rather than helium in the hottest stars $(T>40,000 \mathrm{~K})$. Most stars cooler than $40,000 \mathrm{~K}$ have more or less pure $\mathrm{H}$ atmospheres. However, one question, which has not been resolved, concerned the specific nature of the heavy elements and the role of helium in the hottest white dwarfs. One view of white dwarf evolution requires that $\mathrm{H}$-rich DA stars form by gravitational settling of He from either DAO or He-rich central stars of planetary nebulae. In this case, the youngest (hottest) DA white dwarfs may still contain visible traces of $\mathrm{He}$. Spectroscopic observations now available with $E U V E$ provide a crucial test of these ideas. Analysis of data from the EUVE Guest Observer programme and $E U V E$ public archive allows quantitative consideration of the sources of EUV opacity and places limits on the abundance of He which may be present.

\section{Introduction-The View from EUV Surveys}

Among the most significant problems in the study of hot white dwarf evolution has been the existence of two distinct groups having either $\mathrm{H}$ or $\mathrm{He}$ dominated atmospheres and the possible relationships between them and their proposed progenitors, the diverse types of central stars of planetary nebulae (CPN). While the very hottest H-rich DA white dwarfs outnumber He-rich DOs by a factor 7 (Fleming, Liebert \& Green 1986), the relative number of $\mathrm{H}-$ and He-rich CPN is only about $3: 1$. In addition, there is an apparent absence of He-rich stars in the temperature range $30,000-45,000 \mathrm{~K}$, the so-called DB gap, suggesting that $\mathrm{H}$ - and He-dominated groups are not entirely distinct. Several competing processes can affect the composition of a white dwarf atmosphere. He and heavier elements tend to sink out of the photosphere under the influence of gravity but this can be counteracted by radiation pressure. Convective mixing, accretion or mass loss via a weak wind may also play a significant role.

The first soft X-ray and EUV studies of hot DA white dwarfs made the simplifying assumption that He was the sole photospheric opacity source, although the He in these stars was never identified spectroscopically. However, current evidence indicates that heavier elements have an important role to play. The ROSAT EUV/X-ray sky survey data demonstrated that the hottest stars $(T>40,000 \mathrm{~K})$ must have heavy elements in their atmospheres to explain the observed fluxes, but stars below $40,000 \mathrm{~K}$ seem to have little opacity other than that provided by hydrogen (Barstow et al. 1993). For the hottest stars, it is clear that $\mathrm{He}$ alone is insufficient to account for all the opacity and even heavier elements must be involved. Unfortunately, none of the available data permit 
us to decide whether or not He does play a role in DA atmospheres. Optical and IUE data typically only allow upper limits of a few $\times 10^{-4}$ to be placed on the He content and the broadband photometric ROSAT data do not allow separation of the possible opacity contributions from $\mathrm{He}$ and the heavier elements.

\section{The Role of Helium in DA White Dwarfs}

The availability of EUVE spectroscopy has provided us with an opportunity of resolving some of these issues, in particular searching for He with considerably greater sensitivity than has been possible in the past. EUVE allows spectra to be obtained throughout most of the EUV band, from $\approx 60-700 \AA$, but the crucial region is that of the HeII lyman absorption series between $304 \AA$ and the limit at $228 \AA$.

From an analysis point of view, the white dwarfs studied (drawn from both GO programme and $E U V E$ archive) divide into two distinct groups-those with and those without significant heavy element opacity. EUV spectra of the latter category can be described completely by a synthetic spectrum calculated for an $\mathrm{H}+\mathrm{He}$ model atmosphere, together with a suitable model of the interstellar medium (Rumph, Bowyer, \& Vennes 1994). If heavy elements are present, the stars can only be properly studied using complex line-blanketed models with a comprehensive selection of opacity sources. However, it is possible to study just the region of the spectrum near the He II lines, reproducing the general shape of the spectrum with an $\mathrm{H}+\mathrm{He}$ model plus an exponential roll-off towards short wavelengths. A complete description of our data reduction and analysis techniques has been published elsewhere (Barstow, Holberg, \& Koester 1994a, 1994b, 1995).

During the first year of $E U V E$ spectroscopic observations the signal-to-noise was limited to $\approx \pm 20 \%$ by the detector fixed pattern efficiency variation. Subsequently, this problem has been largely diminished by implementation of the 'dither mode' to average out the fixed pattern and leave a residual efficiency variation of $\pm 5 \%$. Figure 1 shows the complete $E U V E$ spectrum of the EUV brightest DA white dwarf HZ43. Although it has a temperature above $40,000 \mathrm{~K}$, the star is known to have a remarkably pure $\mathrm{H}$ atmosphere. It is well fitted by a pure $\mathbf{H}$ model, within the range of temperature and gravity determined by Napiwotzki et al. (1993) and the systematic uncertainties in the models and instrument calibration, although the best fit does require a finite He abundance of $1.3 \times 10^{-6}$. However, since the shape of the continuum flux is quite sensitive to possible systematic effects in the models and instrument calibration, we are cautious about interpreting this as a real detection of He in HZ43. Concentrating on the region spanned by the HeII Lyman series (Figure 2) allows a $90 \%$ upper limit of $3 \times 10^{-7}$ to be placed on the He abundance (Barstow, Holberg, \& Koester 1995).

Table 1 summarises the He abundance limits determined for several stars, with their effective temperatures, gravities and noting whether or not the observation was dithered. The value obtained for $\mathrm{HZ43}$ is substantially lower than any of the others. However, more importantly it is well below the He abundance predicted by diffusion theory (see Vennes et al. 1988), which should be $\approx 3 \times 10^{-6}$ for a star with the temperature and mass of HZ43. This has important consequences for our understanding of the physical mechanisms that determine the photospheric composition of white dwarfs.

\section{Heavy Elements in DA White Dwarfs}

Recently, Marsh et al. (1995) have extended the analysis of ROSAT observations begun by Barstow et al. (1993) to a much larger sample of stars, including most of the new white dwarfs discovered in the survey. While the general conclusions that may be drawn 


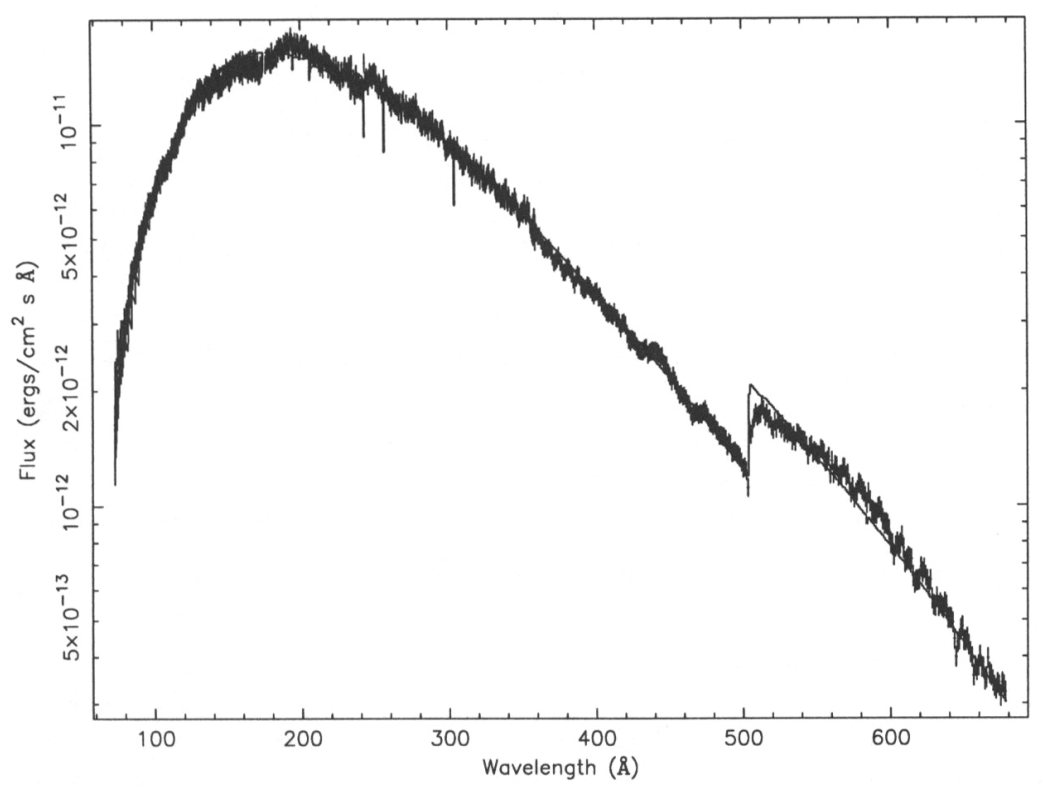

FIGURE 1. Complete EUVE spectrum of the DA white dwarf HZ43 after deconvolution with the instrument response (error bars). The model histogram corresponds to the best fit homogeneous $\mathrm{H}+\mathrm{He}$ model with $T=50,560 \mathrm{~K}, \log g=7.9$, and $\mathrm{He} / \mathrm{H}=1.3 \times 10^{-6}$.

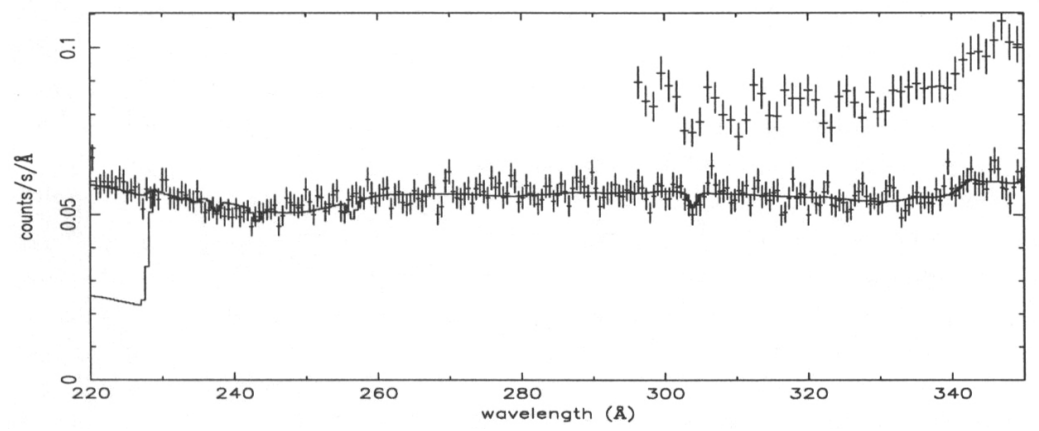

FIGURE 2. Count spectrum of HZ43 between 220 and $310 \AA$, showing a model with $3 \times 10^{-7}$ of photospheric He and demonstrating the size of the interstellar $228 \AA$ edge which would be present if the weak $304 \AA$ "feature" was completely accounted for by an interstellar component. 
TABLE 1. He abundance $90 \%$ upper limits in DA white dwarfs

$\begin{array}{lllcl}\text { Star } & T( \pm 1 \sigma) & \log g( \pm 1 \sigma) & \mathrm{He} / \mathrm{H} & \text { Dithered? } \\ \text { GD659 } & 35,300(500) & 8.00(0.20) & 1.3 \times 10^{-6} & \mathrm{Y} \\ \text { Feige 24 } & 59,800(3,400) & 7.50(0.51) & 3.0 \times 10^{-6} & \mathrm{~N}^{*} \\ \text { GD 71 } & 32,045(148) & 7.72(0.05) & 1.1 \times 10^{-6} & \mathrm{~N} \\ \text { HZ43 } & 49,000(2,000) & 7.70(0.20) & 3.0 \times 10^{-7} & \mathrm{Y}^{* *} \\ \text { RE J2156-546 } & 49,800(3,300) & 7.75(0.18) & 2.0 \times 10^{-6} & \mathrm{~N}^{*} \\ \text { GD246 } & 54,987(931) & 7.71(0.09) & 5.0 \times 10^{-6} & \mathrm{~N}^{*}\end{array}$

** This upper limit is below the level of the He abundance predicted by diffusion theory for a star of this $T$ and $\log g$.

* Close to the predicted value.

TABLE 2. Limits on heavy element abundances in "pure $H$ " DA white dwarfs

HZ43

$\begin{array}{lrccc}\text { Element Abundance } & \text { IUE high res } & \text { EUVE } & \text { IUE high res } & \text { EUVE } \\ \log \mathrm{C} / \mathrm{H} & -8.5 & -5.5 & -7.7 & -4.6 \\ \log \mathrm{N} / \mathrm{H} & -5.0 & -6.0 & -4.2 & -5.4 \\ \log \mathrm{O} / \mathrm{H} & -6.0 & -6.5 & - & -6.0 \\ \log \mathrm{Si} / \mathrm{H} & -8.0 & -6.0 & -9.0 & -7.0 \\ \log \mathrm{Fe} / \mathrm{H} & -4.5 & -4.5 & - & -\end{array}$

remain unaltered, more detail is seen in the $40,000-60,000 \mathrm{~K}$ region. The increase in photospheric opacity is gradual in the $40,000-50,000 \mathrm{~K}$ range but becomes dramatic above $50,000 \mathrm{~K}$. However, it is also apparent that the dispersion in opacities is considerable within a selected narrow temperature range and any theory which seeks to explain the composition of DA white dwarf atmospheres must be able to account for this. EUV spectroscopy can contribute to the solution of this problem through direct study of the sources of EUV opacity in those stars having significant heavy element abundances and measurement of upper limits in stars with near pure $\mathbf{H}$ envelopes.

As an example, upper limits to the abundances of $\mathrm{C}, \mathrm{N}, \mathrm{O}, \mathrm{Si}$, and $\mathrm{Fe}$ have been estimated from the $E U V E$ spectra of HZ43 (T=49,000 K) and GD659 (T=35,300 K; see also Holberg et al. 1995). The strengths of EUV absorption lines were calculated for a range of abundances, using the TLUSTY and SYNSPEC non-LTE codes (Hubeny 1988; Hubeny \& Lanz 1992, 1995), and compared with the data. Any lines with a depth approximately twice the amplitude of the signal-to-noise of the spectra should be readily detected and the upper limits are defined as the abundance at which the strongest features just reach this threshold. These results are summarised in Table 2 and compared with limits, derived in a similar way, for coadded IUE high dispersion spectra. While IUE provides the best constraints on $\mathrm{C}$ and $\mathrm{Si}$, the EUV data are much more sensitive to $\mathrm{N}$ and $\mathrm{O}$. At the effective temperatures in question, NIV and OIV are the most populated ionisation states and most of their bound-bound transitions are found in the EUV rather than the far-UV.

Currently, analysis of EUVE spectra of white dwarfs containing large quantities of 


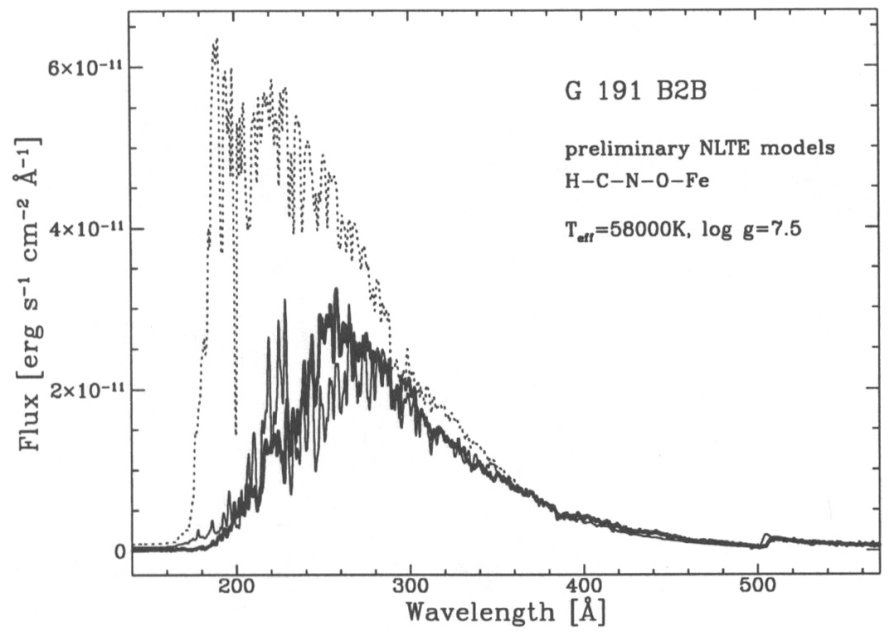

FIGURE 3. EUVE spectrum of G191-B2B (thick solid line) compared with two non-LTE line blanketed models for $T=58,000 \mathrm{~K}, \log g=7.5, \mathrm{C} / \mathrm{H}=2 \times 10^{-6}, \mathrm{~N} / \mathrm{H}=1.75 \times 10^{-7}$, $\mathrm{O} / \mathrm{H}=1.0 \times 10^{-6}$, and $\mathrm{Fe} / \mathrm{H}=6.5 \times 10^{-5}$. Dashed line-6,000 lines; thin solid line-300,000 lines. Interstellar absorption is included with $\mathrm{HI}=2.5 \times 10^{18} \mathrm{~cm}^{-2}$, $\mathrm{HeI} / \mathrm{HI}=0.09$ and $\mathrm{HeII} / \mathrm{HI}=0.01$

heavy elements poses some difficulty. Some progress has been made for objects with less extreme abundances, such as PG1234+482 (see Jordan et al. these proceedings), using LTE models. However, with considerable blanketing from Fe group lines contributing to the overall opacity, non-LTE effects are expected to be important. Unfortunately, non-LTE models of stars like G191-B2B have been completely unable to match the observations whereas LTE models can yield a reasonable representation (see Koester, these proceedings). The problem seems to stem from the difficulties of dealing with a large enough number of heavy element lines in non-LTE. Recent state-of-the-art calculations (Lanz \& Hubeny 1995) have typically used the Kurucz line list, considering only the 36,000 lines between observed levels with some 6,000 contributing to the EUV opacity in the range from 25 to $600 \AA$, but cannot match the steep fall in the spectrum below $250 \AA$ (thin dashed line, Figure 3). Taking all the lines predicted by Kurucz for the higher levels of $\mathrm{FeIV}, \mathrm{FeV}$, and FeVI increases the total number of lines to about 730,000. About 300,000 of these contribute significantly to the opacity and the new calculation yields a large improvement in the level of agreement between model (thin solid line) and data (thick solid line). Reworking of the codes to deal more easily with such large numbers of lines should allow progress to be made in this area in the near future.

\section{The Composition of RE J0503-289}

The hot helium-rich DO white dwarf RE J0503-289 lies in a region of extremely low interstellar hydrogen density (Barstow et al. 1994). As the only DO white dwarf detected 


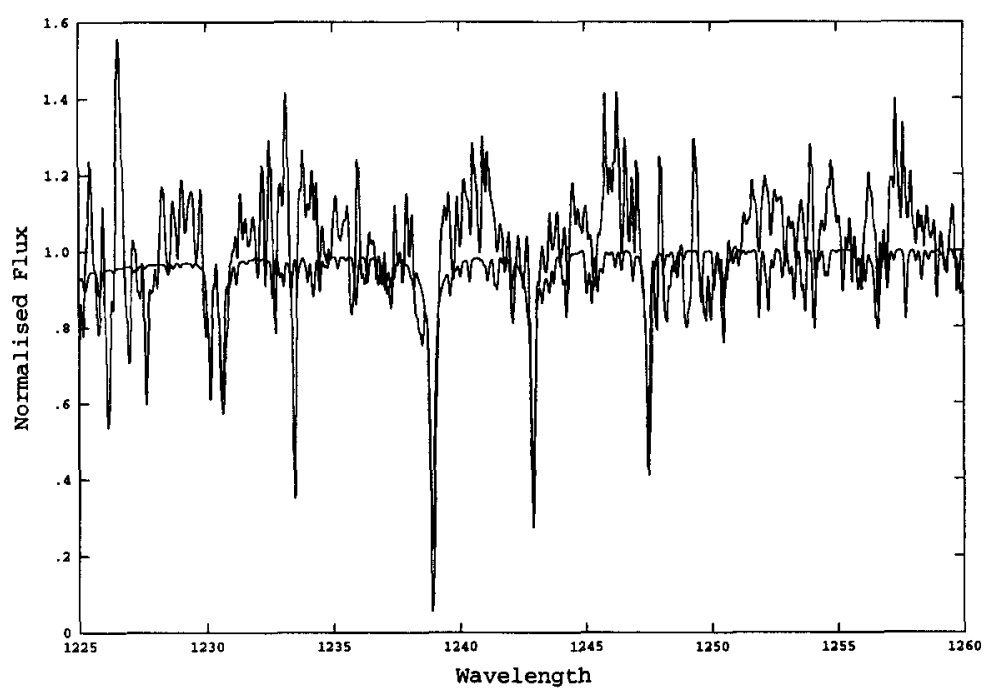

FIGURE 4. Region of the IUE high dispersion spectrum of RE J0503-289 showing NV, CIV and CIII features compared to a $63,000 \mathrm{~K}$ model with $\mathrm{C}: \mathrm{He}=0.1 \%, \mathrm{~N}: \mathrm{He}=0.01 \%, \mathrm{O}: \mathrm{He}=0.01 \%$, $\mathrm{Si}: \mathrm{He}=0.01 \%$ and $\mathrm{Ni}: \mathrm{He}=0.001 \%$.

in both ROSAT WFC and EUVE sky surveys, it is a very important target for further study with the EUVE spectrometer. The star is also bright enough to be observed at high dispersion with $I U E$ and we have acquired four spectra during the past two years. Detection of weak P Cygni profiles in one of these is evidence for the presence of an episodic weak wind in this object (Barstow \& Sion 1994).

The IUE and EUVE data show that, in addition to the carbon already discovered in our earlier optical and low dispersion $I U E$ observations, the atmosphere of the star contains significant quantities of nitrogen, oxygen and silicon. There is no sign of any $\mathrm{Fe}$, found in DA white dwarfs of similar temperature, but many of the weaker features in the spectrum coincide with Ni V transitions. More detailed analysis of the photospheric composition of RE J0503-289 has proved difficult. It is possible to estimate photospheric abundances by comparing non-LTE model spectra with the measured UV line strengths. However, the EUV fluxes predicted by the same models which match the UV data are up to an order of magnitude greater than observed. In an attempt to reconcile the EUV and far-UV data, we constructed a grid of non-LTE spectra for a range of temperature and composition and fit both spectral ranges simultaneously. A consistent solution is achieved with a temperature of $63000 \mathrm{~K}$ (cf. 70000 from earlier work) and a $\mathrm{C}$ abundance of $0.1 \%$, a factor 10 below that originally estimated. Abundances of $\mathrm{N}, \mathrm{O}$, and $\mathrm{Si}$ are all about $0.01 \%$ and that of $\mathrm{Ni} 0.001 \%$, with respect to helium. Figure 4 shows a section of the IUE spectrum spanning the wavelength range from 1225 to $1260 \AA$, including N V, C IV and CIII features compared to a non-LTE model computed for these abundances. The EUV model spectrum is now a very good match to the data (Figure 5), although the predicted $\mathrm{He}$ II line profiles are rather broader than observed. This probably arises from the absence of a satisfactory broadening theory for the He II Lyman series. We hope to address this problem with theorists working on the atomic data. 


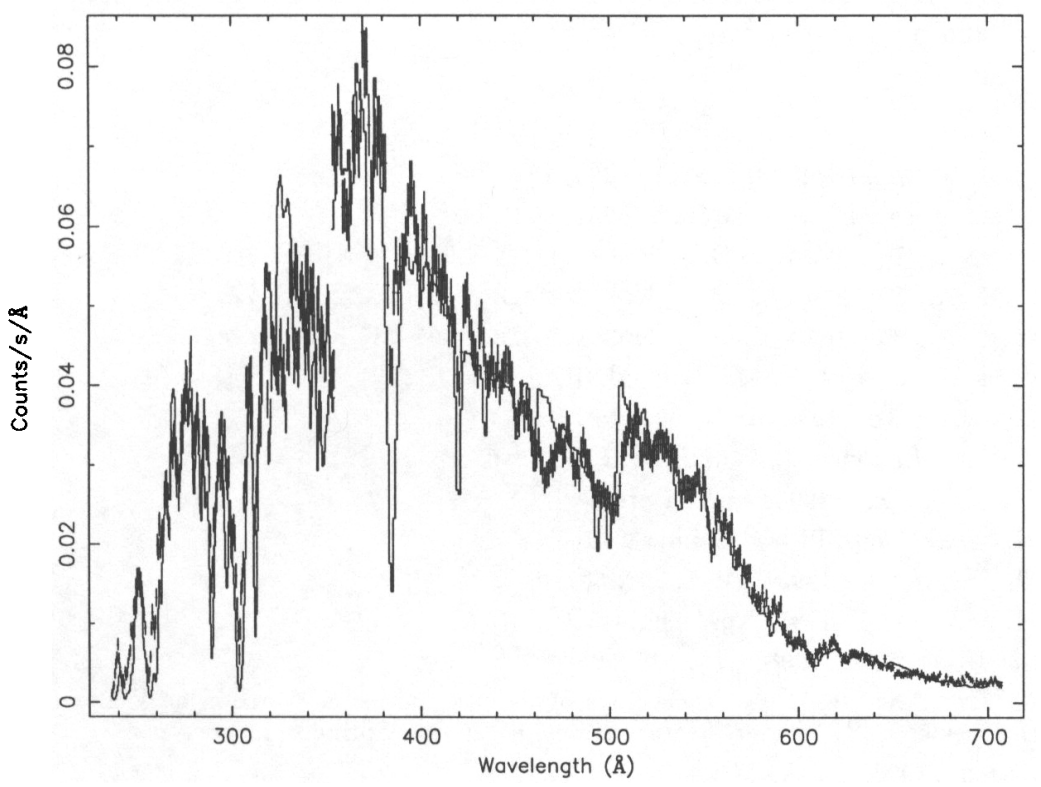

FiguRE 5. EUVE spectrum of RE J0503-289 compared to the best fit model discussed in the text.

\section{Conclusion}

It is clear that EUV spectroscopy is already making significant contributions to our understanding of white dwarf atmospheres. For example, important progress has been made in understanding the role of helium in DA stars. Limits placed on the He abundance of a number of typical DAs, spanning a temperature range from 30,000 to $60,000 \mathrm{~K}$ are 2 to 3 orders of magnitude below the values typical of DAO white dwarfs. Furthermore, the allowed abundances of $\mathrm{He}$ are below a level where the Balmer line temperature measurement becomes problematic. In those DA stars which have low levels of heavy element abundances in their atmospheres, the EUVE spectra can also be used to place limit on their abundances. The higher spectral resolution of the IUE echelle gives the best limits on carbon and silicon but the most populated ions of nitrogen and oxygen, NIV and OIV, have their strongest transitions in the EUV. Hence, EUVE data give more stringent limits on these elements.

Study of the very hottest DA white dwarfs such as G191-B2B, which contain significant quantities of heavy elements, has proved difficult because it has not been possible to calculate detailed model spectra which can match even the general shape of the spectral data. However, it seems that this is a result of not including sufficient numbers of detailed line transitions, mainly for iron ions, in the non-LTE calculations. We hope that imminent improvements in the computer programmes will solve this problem. In contrast, we have been able to make significant progress in understanding the atmosphere of the DO white dwarf RE J0503-289, which contains significant quantities of carbon, nitrogen, oxygen, and silicon but shows no evidence for iron. 
MAB acknowledges the support of PPARC, UK. The work of IH and TL was supported in part by NASA grant NAGW-3834 and that of EMS by NASA LTSA grant NAGW5716 to Villanova University.

\section{REFERENCES}

Barstow, M. A. et AL. 1993, MNRAS, 264, 16

BARSTOW, M. A. ET AL. 1994, MNRAS, 267, 653

Barstow, M. A., Holberg, J. B. \& Koester, D. 1994a, MNRAS, 268, L35

Barstow, M. A., Holberg, J. B. \& Koester, D. 1994b, MNRAS, 270, 516

Barstow, M. A., Holberg, J. B. \& Koester, D. 1995, MNRAS, in press

BARstow, M. A. \& SiON, E. M. 1994, MNRAS, 271, L52

Bergeron, P., et AL. 1994, ApJ, 432, 305

Fleming, T. A., Liebert, J. \& Green, R. F. 1986, ApJ, 308, 176

Holberg, J. B. ET AL. 1995, ApJ, in press

HUBENY, I. 1988, Comp. Phys. Comm., 52, 103

Hubeny, I. \& Lanz, T. 1992, A\& A, 262, 501

Hubeny, I. \& Lanz, T. 1995, ApJ, 439, 875

LANZ, T. \& HuBENY, T. 1995, ApJ, 439, 905

Marsh, M. C. Et AL. 1995, in Proceedings of the 9th European Worshop on White Dwarfs, ed. D. Koester \& K. Werner, Lecture Notes in Physics, Springer, in press

NAPIWOTZKI, R. 1993, A\& A, 278, 478

Rumph, T., Bowyer, S. \& VenNes, S. 1994, AJ, 107, 2108

Vennes, S., Pelletier, C., Fontaine, G., \& Wesemael, F. 1988, ApJ, 331, 876 